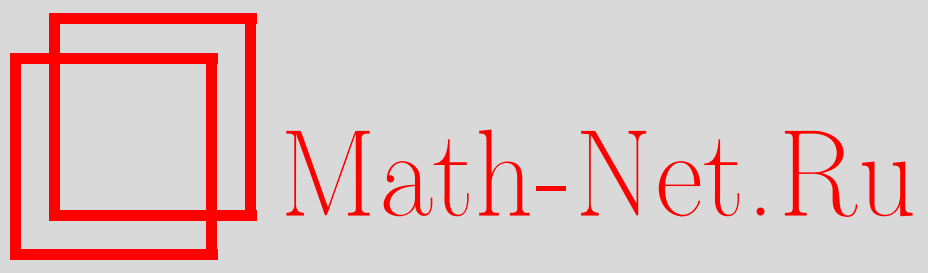

Л. Д. Фаддеев, Дискретная серия представлений для модулярного дубля квантовой группы $U_{q}(\operatorname{sl}(2, \mathbb{R}))$, Функи. анализ и его прил., 2008, том 42, выпуск 4, 98-104

DOI: https://doi.org/10.4213/faa2927

Использование Общероссийского математического портала MathNet.Ru подразумевает, что вы прочитали и согласны с пользовательским соглашением

http://www . mathnet.ru/rus/agreement

Параметры загрузки:

IP: 54.197 .130 .99

26 апреля 2023 г., 14:37:21

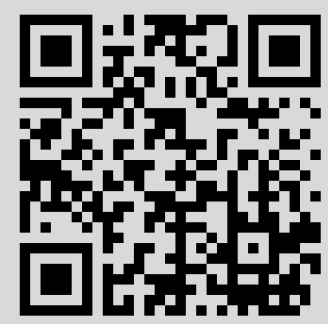


Функииональный анализ и его приложения

2008, т. 42, вып. 4, с. 98-104

УДК $517.98+512.54$

\title{
Дискретная серия представлений для модулярного дубля квантовой группы $U_{q}(\operatorname{sl}(2, \mathbb{R}))$
}

\author{
(c) 2008. Л. Д. ФАДДЕЕВ*
}

Дорогому Израилю Моисеевичу Гельфанду

\section{§1. Введение}

Модулярный дубль квантовой группы $U_{q}(\operatorname{sl}(2))$ - это, грубо говоря, тензорное произведение двух квантовых групп с параметрами деформации $q=e^{i \pi \tau}$ и $\tilde{q}=e^{i \pi / \tau}$. Этот объект, предложенный мной в [1], был открыт в результате поиска регуляризации универсальной $R$-матрицы для корней из 1 . Его значение для конформной теории поля, в особенности с некомпактными полевыми переменными (например, модели Лиувилля), довольно очевидно. В серии работ [2]-[5] использовалась непрерывная серия унитарных представлений модулярного дубля некомпактной вещественной формы $U_{q}(\mathrm{sl}(2, \mathbb{R}))$, параметризованная положительным числом - спином. Было показано, что эти представления не разлагаются в тензорные произведения дуальных факторов.

Представления были построены для двух интервалов параметра деформации:

режим I: $\tau>0,|q|=|\tilde{q}|=1$;

режим II: $|\tau|=1, \bar{q}=\tilde{q}$.

В этой работе мы предъявим еще одну серию представлений, которая существует только для режима II. Эта серия параметризуется двумя целыми числами $n=1,2, \ldots, m=1, \ldots, n$.

Во втором параграфе мы напоминаем известные результаты, упомянутые выше, а в третьем описываем новые представления.

Я благодарен П. П. Кулишу и М. А. Семенову-Тян-Шанскому за важные замечания.

\section{§2. Разминка - непрерывная серия}

Набор генераторов модулярного дубля для $U_{q}(\mathrm{sl}(2))$ состоит из двух троек $E, F, K$ и $\widetilde{E}, \widetilde{F}, \widetilde{K}$, удовлетворяющих соотношениям

$$
\begin{aligned}
K E & =q^{2} E K, \\
K F & =q^{-2} F K, \\
E F-F E & =\frac{1}{q-q^{-1}}\left(K-K^{-1}\right)
\end{aligned}
$$

*Работа частично поддержана грантом РФФИ 08-01-00638 и программой «Математические проблемы нелинейной динамики» Президиума РАН. 
с комплексным параметром $q=e^{i \pi \tau}$ и аналогичным соотношениям для $\widetilde{E}, \widetilde{F}$, $\widetilde{K}$ с параметром $\tilde{q}=e^{i \pi / \tau}$.

Отметим, что мы используем дуальный параметр $1 / \tau$ вместо более традиционного $-1 / \tau$. Эта подмена не принципиальна и обусловлена только соображениями удобства.

Генераторы $E, F, K$ коммутируют с генераторами $\widetilde{E}, \widetilde{F}, \widetilde{K}$. Мы будем использовать выражение для этих генераторов через более простые переменные по аналогии с переменными Гельфанда-Кириллова для некомпактной универсальной обертывающей алгебры $U(\operatorname{sl}(2))$. Пусть $u, v$ - пара Вейля с соотношением $u v=q^{2} v u$ и $Z$ - центральный элемент. Тогда

$$
E=i \frac{v+u^{-1} Z}{q-q^{-1}}, \quad F=i \frac{u+v^{-1} Z^{-1}}{q-q^{-1}}, \quad K=q^{-1} u v
$$

удовлетворяют соотношениям (1)-(3). Традиционный центральный элемент

$$
C=q K+q^{-1} K^{-1}+\left(q-q^{-1}\right)^{2} F E
$$

выражается через $Z$ следующим образом:

$$
C=-\left(Z+Z^{-1}\right)
$$

В [3]-[5] использовалась аналогичная и эквивалентная параметризация.

Дуальные генераторы выражаются аналогичным образом через $\tilde{u}, \tilde{v}, \tilde{Z}$, где

$$
\tilde{u}=u^{1 / \tau}, \quad \tilde{v}=v^{1 / \tau}, \quad \widetilde{Z}=Z^{1 / \tau} .
$$

Тот факт, что $E, F, K$ коммутируют с $\widetilde{E}, \widetilde{F}, \widetilde{K}$, обеспечивается формулой Эйлера $e^{2 \pi i}=1$. Представления [3], [4] реализованы в $L_{2}(\mathbb{R})$, где пара Вейля действует как умножение и сдвиг. Нам удобно использовать вейерштрассовы параметры $\omega, \omega^{\prime}$ вместо $\tau$,

$$
\omega \omega^{\prime}=-\frac{1}{4}, \quad \tau=\frac{\omega^{\prime}}{\omega} ;
$$

переход к дуальному параметру $1 / \tau$ соответствует перестановке $\omega$ и $\omega^{\prime}$.

Операторы $u, v$ действуют на элемент $f(x), x \in \mathbb{R}$, из $L_{2}(\mathbb{R})$ следующим образом:

$$
\begin{aligned}
& u f(x)=e^{-i \pi x / \omega} f(x), \\
& v f(x)=f\left(x+2 \omega^{\prime}\right) .
\end{aligned}
$$

Сдвиг $x \mapsto x+2 \omega^{\prime}$ уводит нас с вещественной оси, и функция $e^{-i \pi x / \omega}$ неограничена при вещественных $x$; таким образом, мы должны указать область определения для $u$ и $v$. Будем считать, что плотная область определения состоит из целых функций $f(x), x \in \mathbb{C}$, быстро убывающих вдоль контуров, параллельных вещественной оси. Эта область содержит функции вида $f(x)=e^{-x^{2}} P(x)$, где $P(x)$ - полином.

При таком соглашении легко сосчитать оператор, сопряженный с $v$ по отношению к обычному скалярному произведению в $L_{2}(\mathbb{R})$

$$
(f, g)=\int_{-\infty}^{\infty} \overline{f(x)} g(x) d x .
$$


Действительно,

$$
\begin{aligned}
(f, v g) & =\int_{-\infty}^{\infty} \overline{f(x)} g\left(x+2 \omega^{\prime}\right) d x=\int_{-\infty}^{\infty} \bar{f}(x) g\left(x+2 \omega^{\prime}\right) d x \\
& =\int_{-\infty+2 \omega^{\prime}}^{\infty+2 \omega^{\prime}} \bar{f}\left(x-2 \omega^{\prime}\right) g(x) d x=\int_{-\infty}^{\infty} \overline{f\left(x-\overline{2 \omega^{\prime}}\right)} g(x) d x=\left(v^{*} f, g\right),
\end{aligned}
$$

где $v^{*} f(x)=f\left(x-\overline{2 \omega^{\prime}}\right)$. В этой выкладке мы обозначили через $\bar{f}(x)$ аналитическую функцию от $x$, коэффициенты которой при разложении по степеням $x$ комплексно сопряжены коэффициентам для $f$.

Мы можем теперь ввести вещественную форму для нашей алгебры. Для этого есть две возможности выбора $\tau$, которые мы назовем режимом I и режимом II.

Режим I: $\tau-$ положительное число, $\omega$ и $\omega^{\prime}$ чисто мнимые,

$$
\bar{\omega}=-\omega, \quad \bar{\omega}^{\prime}=-\omega^{\prime} .
$$

Операторы $u, v$ и $\tilde{u}, \tilde{v}$ эрмитовы и положительно определенные,

$$
u^{*}=u, \quad v^{*}=v, \quad \tilde{u}^{*}=\tilde{u}, \quad \tilde{v}^{*}=\tilde{v} .
$$

Это же верно и для $E, F, K$ и $\widetilde{E}, \widetilde{F}, \widetilde{K}$, если $Z$ эрмитов. В неприводимом представлении $Z$ и $\widetilde{Z}$ являются вещественными числами. Мы задаем их как

$$
Z=e^{i \pi a / \omega}, \quad \widetilde{Z}=e^{i \pi a / \omega^{\prime}},
$$

где $a$ - вещественный параметр - спин. В [3]-[5] приведены все необходимые математические детали.

Режим II: $|\tau|=1$,

$$
\bar{\omega}=-\omega^{\prime} .
$$

Операторы $u, v$ эрмитово сопряжены к $\tilde{u}, \tilde{v}$,

$$
u^{*}=\tilde{u}, \quad v^{*}=\tilde{v},
$$

и то же верно для чисел $Z$ и $\widetilde{Z}$, если мы используем параметризацию (7) с вещественным $a$.

Заметим, что третий параметр Вейерштрасса

$$
\omega^{\prime \prime}=\omega+\omega^{\prime}
$$

является чисто мнимым в обоих режимах.

На этом мы закончим напоминание известных вещей. Ясно, что скалярное произведение (6) использовано исчерпывающим образом. В следующем параграфе мы рассмотрим другую возможность.

\section{§3. Дискретная серия}

Мы будем использовать другое скалярное произведение для функций $f$, на которые действуют вейлевские операторы. Теперь это аналитические функции $f(z)$ и действие операторов $u, v, \tilde{u}, \tilde{v}$ такое же, как в (4), (5), с заменой $x \mapsto z$. Скалярное произведение дается формулой

$$
\langle f, g\rangle=\int_{D} \overline{f(z)} S(\bar{z}, z) g(z) \frac{d z d \bar{z}}{2 \pi i},
$$

где интегрирование ведется по некоторой области $D$ на комплексной плоскости. Ядро $S(\bar{z}, z)$ должно быть положительным. 
Мы найдем такое ядро и, тем самым, представление в режиме II для дискретных значений спина $a$, а именно $a=-n \omega^{\prime \prime}$, где $n-$ положительное число. Такой спин - чисто мнимое число, и условие вещественности представления, в частности, требует соотношения

$$
\widetilde{Z}=Z^{-1}
$$

Начнем с соотношения

$$
\widetilde{K}^{*}=K
$$

Мы имеем

$$
\begin{aligned}
\langle\widetilde{K} f, g\rangle & =\int \overline{\tilde{q}^{-1} e^{-i \pi z / \omega^{\prime}} f(z+2 \omega)} S(\bar{z}, z) g(z) \frac{d z d \bar{z}}{2 \pi i} \\
& =q \int \bar{f}\left(\bar{z}-2 \omega^{\prime}\right) S(\bar{z}, z) e^{-i \pi \bar{z} / \omega} g(z) \frac{d z d \bar{z}}{2 \pi i} \\
& =q^{-1} \int \overline{f(z)} S\left(\bar{z}+2 \omega^{\prime}, z\right) e^{-i \pi \bar{z} / \omega} g(z) \frac{d z d \bar{z}}{2 \pi i}
\end{aligned}
$$

и

$$
\begin{aligned}
\langle f, K g\rangle & =\int \overline{f(z)} S(\bar{z}, z) q^{-1} e^{-i \pi z / \omega} g\left(z+2 \omega^{\prime}\right) \frac{d z d \bar{z}}{2 \pi i} \\
& =q \int \overline{f(z)} S\left(\bar{z}, z-2 \omega^{\prime}\right) e^{-i \pi z / \omega} g(z) \frac{d z d \bar{z}}{2 \pi i} .
\end{aligned}
$$

Таким образом, мы приходим к уравнению

$$
q S\left(\bar{z}, z-2 \omega^{\prime}\right) e^{-i \pi z / \omega}=q^{-1} S\left(\bar{z}+2 \omega^{\prime}, z\right) e^{-i \pi \bar{z} / \omega},
$$

которое удовлетворяется, если $S(\bar{z}, z)$ представляется в виде

$$
S(\bar{z}, z)=\exp i \pi\left(z^{2}-\bar{z}^{2}\right) \Phi(\bar{z}-z) .
$$

Обратимся теперь к условию $E^{*}=\widetilde{E}$. Мы имеем

$$
\begin{aligned}
\langle f, E g\rangle & =\frac{i}{q-q^{-1}} \int \overline{f(z)} S(\bar{z}, z)\left(g\left(z+2 \omega^{\prime}\right)+Z e^{i \pi z / \omega} g(z)\right) \frac{d z d \bar{z}}{2 \pi i} \\
& =\frac{i}{q-q^{-1}} \int \overline{f(z)}\left(S\left(\bar{z}, z-2 \omega^{\prime}\right)+Z S(\bar{z}, z) e^{i \pi z / \omega}\right) g(z) \frac{d z d \bar{z}}{2 \pi i}
\end{aligned}
$$

и

$$
\begin{aligned}
(\widetilde{E} f, g) & =\int \frac{i}{\frac{i}{\tilde{q}-\tilde{q}^{-1}}\left(f(z+2 \omega)+Z e^{i \pi z / \omega^{\prime}} f(z)\right)} S(\bar{z}, z) g(z) \frac{d z d \bar{z}}{2 \pi i} \\
& =\frac{i}{q-q^{-1}} \int \overline{f(z)}\left(S\left(\bar{z}+2 \omega^{\prime}, z\right)+\overline{\widetilde{Z}} S(\bar{z}, z) e^{i \pi \bar{z} / \omega}\right) g(z) \frac{d z d \bar{z}}{2 \pi i},
\end{aligned}
$$

что приводит к уравнению

$$
S\left(\bar{z}, z-2 \omega^{\prime}\right)+Z e^{i \pi z / \omega} S(\bar{z}, z)=S\left(\bar{z}+2 \omega^{\prime}, z\right)+Z^{-1} e^{i \pi \bar{z} / \omega} S(\bar{z}, z) .
$$

Используя (9), приходим к уравнению для $\Phi(t), t=\bar{z}-z$,

$$
\left(q^{-1} e^{-i \pi t / \omega}-q\right) \Phi\left(t+2 \omega^{\prime}\right)=\left(Z^{-1}-Z e^{-i \pi t / \omega}\right) \Phi(t) .
$$


Подставляя $Z=e^{-i \pi a / \omega}$ (замена знака будет полезна ниже) и замечая, что $q=e^{i \pi \omega^{\prime} / \omega}=-e^{i \pi \omega^{\prime \prime} / \omega}$, мы можем переписать последнее уравнение в виде

$$
\frac{\Phi\left(t+2 \omega^{\prime}\right)}{\Phi(t)}=\frac{\sin \pi \frac{t+2 a}{2 \omega}}{\sin \pi \frac{t+2 \omega^{\prime \prime}}{2 \omega}} .
$$

Условие

$$
(\widetilde{F} f, g)=(f, F g)
$$

приводит к такому же уравнению. Условия

$$
(E f, g)=(f, \tilde{E} g), \quad(F f, g)=(f, \widetilde{F} g)
$$

приводят к аналогичному уравнению с перестановкой $\omega$ и $\omega^{\prime}$ :

$$
\frac{\Phi(t+2 \omega)}{\Phi(t)}=\frac{\sin \pi \frac{t+2 a}{2 \omega^{\prime}}}{\sin \pi \frac{t+2 \omega^{\prime \prime}}{2 \omega^{\prime}}} .
$$

Уравнения (10) и (11) фиксируют $\Phi(t)$ однозначно с точностью до постоянного множителя.

Мы будем искать решение $\Phi(t)$, положительное при мнимом $t$. Легко проверить, что для дискретных значений спина $a$,

$$
a=n \omega^{\prime \prime}, \quad n=1,2, \ldots,
$$

таковы решения $\Phi(t)=1$ при $n=1$ и

$$
\Phi(t)=\prod_{m=1}^{n-1} \sin \pi \frac{t+2 m \omega^{\prime \prime}}{2 \omega^{\prime}} \sin \pi \frac{t+2 m \omega^{\prime \prime}}{2 \omega}, \quad n=2,3, \ldots .
$$

Для мнимых $t$ это выражение может быть переписано как

$$
\Phi(t)=\prod_{m=1}^{n-1}\left|\sin \pi \frac{t+2 m \omega^{\prime \prime}}{2 \omega^{\prime}}\right|^{2}
$$

и, таким образом, явно неотрицательно.

Линии нулей функции $\Phi$

$$
t=-2 m \omega^{\prime \prime}
$$

делят всю комплексную плоскость на $n$ частей: две полуплоскости $y<\mu$ и $y>\mu n$ и $n-2$ полос конечной ширины

$$
\mu m<y<\mu(m+1), \quad m=1, \ldots n-2,
$$

где

$$
y=\frac{z-\bar{z}}{2 i}, \quad \mu=\frac{\omega^{\prime \prime}}{i} .
$$

Каждую из этих частей мы возьмем в качестве области интегрирования в (8). Следует думать, что соответствующие представления неэквивалентны. Если это так, то мы имеем серию представлений, параметризованных дискретным спином из (12) и дополнительным числом $m=1, \ldots, n-1$. Оставляем это утверждение в виде гипотезы.

Для общего значения мнимого спина $a$ решение уравнений (10) и (11) можно выразить через «некомпактный квантовый дилогарифм», ясно описанный в [7] и уже зарекомендовавший себя как важнейший объект конформной теории 
поля и квантовой теории Тейхмюллера [8], [9]. Эта функция $\gamma(\zeta)$ определяется дуальными функциональными уравнениями

$$
\begin{aligned}
& \frac{\gamma\left(\zeta+\omega^{\prime}\right)}{\gamma\left(\zeta-\omega^{\prime}\right)}=1+e^{-i \pi \zeta / \omega} \\
& \frac{\gamma(\zeta+\omega)}{\gamma(\zeta-\omega)}=1+e^{-i \pi \zeta / \omega^{\prime}}
\end{aligned}
$$

Решение $\Phi(t)$ может быть явно записано как

$$
\Phi(t)=e^{2 \pi i\left(a+\omega^{\prime \prime}\right)} \frac{\gamma\left(t-\omega^{\prime \prime}+2 a\right)}{\gamma\left(t+\omega^{\prime \prime}\right)} .
$$

При $a=n \omega^{\prime \prime}(16)$ сводится к (13) при помощи соотношения

$$
\frac{\gamma\left(\zeta+\omega^{\prime \prime}\right)}{\gamma\left(\zeta-\omega^{\prime \prime}\right)}=-4 e^{2 \pi i \zeta \omega^{\prime \prime}} \sin \frac{\pi \zeta}{2 \omega^{\prime}} \sin \frac{\pi \zeta}{2 \omega},
$$

которое следует из (14), (15). Я считаю, что (16) имеет положительные значения при мнимых $t$ только при $a=n \omega^{\prime \prime}$.

Стоит заметить связь параметров $n$ и $m$ с нулями функции $\gamma(z)$ на верхней полуплоскости, которые даются формулой

$$
z_{p, q}=\omega^{\prime \prime}+2 p \omega+2 q \omega^{\prime}
$$

где $p, q$ - целые неотрицательные числа. Ясно, что на каждом уровне $\operatorname{Im} z=$ $n \omega^{\prime \prime}$ мы имеем $n$ нулей в полном соответствии с числом представлений. Это заставляет нас думать о деформированной формуле Планшереля. Мы оставляем это для дальнейшей работы.

\section{§4. Заключение}

Представления, найденные здесь, имеют смысл только в режиме II. Действительно, если мы повторим вычисление для режима I, то получим то же уравнение для $\Phi(t)$, однако аргументы синусов станут вещественными при мнимом $t$ и $\Phi(t)$ будет иметь бесконечное число нулей.

Я считаю, что режим II интереснее, чем режим I, как математически, так и в физических приложениях. Действительно, в режиме II числа $\omega, \omega^{\prime}$ образуют решетку в $\mathbb{C}$, в то время как в режиме I они вырожденно лежат на мнимой оси. В модели Лиувилля центральный заряд алгебры Вирасоро задается формулой

$$
C=1+6\left(\tau+\frac{1}{\tau}+2\right)
$$

(см. [9], [10]), так что $c>25$ в режиме I и $1<c<25$ в режиме II. Именно последний интервал интересен для приложения модели Лиувилля к некритической струне Полякова [11]. Найденные здесь представления открывают возможности для поиска квантования квантовой модели Лиувилля, которое отличается от простого продолжения формул, полученных при $c>25$ [12]. Отметим еще одно обстоятельство. В недеформированном случае дискретную серию имеет только группа $S L(2, \mathbb{R})$, но не ее алгебра Ли. В нашем случае алгебра $U_{q}(s l(2, \mathbb{R}))$ соответствует деформированной алгебре Ли, которая, таким образом, похожа более на группу Ли, чем на ее инфинитезимальную алгебру. 


\section{ЛитерАТУРА}

[1] L. D. Faddeev, Modular double of a quantum group, in: Math. Phys. Studies, vol. 21, Kluwer Acad. Publ., Dordrecht, 2000, 149-156.

[2] A. G. Bytsko, J. Teschner, Quantization of models non-compact quantum group symmetry: modular XXZ magnet and lattice sinh-Gordon model, J. Phys. A: Math. Gen., 39:41 (2006), 12927-12981.

[3] B. Ponsot, J. Teschner, Clebsch-Gordan and Racah-Wigner coefficients for a continuous series of representations of $U_{q}(\mathrm{sl}(2, \mathbb{R}))$, Comm. Math. Phys., 224:3 (2001), 613-655.

[4] S. Kharchev, D. Lebedev, M. Semenov-Tian-Shansky, Unitary representations of $U_{q}\left(\mathrm{sl}_{2}(\mathbb{R})\right)$, the modular double, and the multiparticle $q$-deformed Toda chains, Comm. Math. Phys., 225:3 (2002), 573-609.

[5] K. Schmüdgen, Operator representation of $U_{q}(\operatorname{sl}(2))$, Lett. Math. Phys., $37: 2$ (1996), 211-222.

[6] A. Yu. Volkov, Noncommutative hypergeometry, Comm. Math. Phys., 258:2 (2005), $257-273$.

[7] R. M. Kashaev, Quantization of Teichmüller spaces and the quantum dilogarithm, Lett. Math. Phys., 43:2 (1998), 105-115.

[8] В. В. Фок, Л. О. Чехов, Квантовые пространства Тейхмюллера, ТМФ, 120:3 (1999), 511-528.

[9] J.-L. Gervais, A. Neveu, The dual string spectrum in Polyakov's quantization, I, Nucl. Phys. B, 199:1 (1982), 59-76.

[10] T. L. Curtright, C. B. Thorn, Conformally invariant quantization of the Liouville theory, Phys. Rev. Lett., 48:19 (1982), 1309-1313.

[11] A. M. Polyakov, Quantum geometry of bosonic strings, Phys. Lett. B, 103:3 (1981), 207-210.

[12] V. V. Bazhanov, S. L. Lukyanov, A. B. Zamolodchikov, Integrable structure of conformal field theory, quantum KdV theory and thermodynamic Bethe ansatz, Comm. Math. Phys., 177:2 (1996), 381; http://arxiv.org/abs/hep-th/9412229.

Санкт-Петербургское отделение Математического института им. В. А. Стеклова РАН

Поступило в редакцию e-mail: faddeev@pdmi.ras.ru 